\title{
LARVAS DE MYRMELEON BRASILIENSIS (NEUROPTERA, MYRMELEONTIDAE] COMO MODELO PARA ESTUDO DO COMPORTAMENTO DE PREDAÇÃO
}

\author{
LARVAE OF MYRMELEON BRASILIENSIS [NEUROPTERA, MYRMELEONTIDAE] \\ AS MODEL FOR STUDY OF PREDATION BEHAVIOR
}

\author{
Aleksander Ribeiro Hada \\ Fabíola da Costa Catombé Dantas ${ }^{2}$ \\ Guilherme Santos Toledo de Lima ${ }^{3}$ \\ Talita Ferreira Amado ${ }^{4}$
}

\section{Resumo}

Predadores de emboscada investem muita energia na construção de armadilhas para captura de presas. Esse é o caso das larvas de formiga-leão, que acessam seus recursos alimentares por meio da construção funis de areia. O objetivo pricipal deste estudo foi verificar se o tamanho da larva do predador (formiga-leão) e a probabilidade de predação estão relacionados ao tamanho da armadilha. O experimento foi conduzido na Estação Ecológica do Seridó, RN, durante o mês de maio de 2012. Foram demarcados 12 quadrantes de $1 \mathrm{~m}^{2}$ ao longo de dois transectos de $40 \mathrm{~m}$. Em cada quadrante foram escolhidos aleatoriamente 4 armadilhas, sendo uma de cada classe de tamanho, onde eram lançadas as presas (térmitas) nas armadilhas. Nós medimos o tempo de captura da presa, comprimentos das larvas de formiga-leão e da sua respectiva armadilha. Verificamos que maiores comprimentos de larvas de formiga-leão estão relacionadas a armadilhas também de maior tamanho, mas isso não reflete necessariamente em maior probabilidade de predação de térmitas, o que pode ser explicado pelo conceito de forrageio ótimo. A resposta positiva da probabilidade de predação em função do tamanho da armadilha deve ser verificada quando o tamanho das presas possam oferecer recursos alimentares com ganhos energéticos.

Palavras-chave: formiga-leão, forrageamento ótimo, caatinga.

\begin{abstract}
Ambush predators invests lots of energy to build traps to capture their preys. This is the case of the antlion larvae, that access their food resources through the construction of sand traps. The objective of this study was to determine whether the size of the larva of the predator and the likelihood of predation are related to

1 Instituto de Desenvolvimento Sustentável e Meio Ambiente (IDEMA-RN), Natal, RN, Brasil. E-mail: alekshada@gmail.com

2 Programa de Pós-Graduação em Ecologia, Universidade Federal do Rio Grande do Norte (UFRN), Natal, RN, Brasil. E-mail: fabiola_eco@yahoo.com.br

3 Programa de Pós-Graduação em Ecologia, Universidade Federal do Rio Grande do Norte (UFRN), Natal, RN, Brasil. E-mail: toledolimagui@gmail.com

4 Programa de Pós-Graduação em Ecologia, Universidade Federal do Rio Grande do Norte (UFRN), Natal, RN, Brasil. E-mail: amadotalita@gmail.com
\end{abstract}


the size of the trap. The experiment was conducted in the Seridó Ecological Station/ $\mathrm{RN}$, during the month of May in 2012. 12 quadrats, $1 \mathrm{~m}^{2}$ each, were marked along two transects of $40 \mathrm{~m}$. In each quadrant 4 traps were randomly chosen, one from each size class, then, preys (termites) were released in the traps. The time of capture was measured, in addition, the lengths of ant lion larvae and their respective trap. We found that longer lengths of larval ant lion traps are also related to the larger body size, but this does not necessarily reflect a greater likelihood of predation of termites, which can be explained by the concept of optimal foraging. The positive response of the probability of predation on the size of the trap should be checked when the size of prey can provide food resources with energy gains.

Key words: antlion, optimal foraging, caatinga.

\section{INTRODUÇÃO}

Os organismos utilizam os recursos alimentares para realizarem funções vitais e comportamentais, como manutenção do metabolismo, crescimento, forrageio, construção de abrigos e armadilhas (REYNOLDS, 1990). Cada uma dessas funções possui diferentes níveis de investimento energético, que estão relacionados com a quantidade de recursos alimentares que o indivíduo consome (REYNOLDS et al., 1985).

A teoria do forrageamento ótimo prevê que existe um balanço entre obtenção e gasto de energia durante a procura e manuseio do alimento (TOWNSEND et al., 2003). Para minimizar esses gastos, os organismos apresentam comportamentos de forrageio que otimizam o retorno energético com um mínimo de esforço despendido (BEGON et al., 2006). Para evitar injúrias com a presa e até diminuir a chance de serem predadas, algumas espécies predadoras utilizam a estratégia de "senta-eespera", onde permanecem imóveis, entocadas ou não, aguardando a aproximação de uma presa até capturá-la (RICKLEFS, 2010).

Predadores de emboscada investem muita energia na construção de uma armadilha para captura de presas, entretanto, poupam energia ao evitar o alto custo envolvido na sua procura (LUCAS, 1985; TOWNSEND et al., 2003). As larvas de neurópteros e suas armadilhas são excelentes modelos de estudo de predação por consumirem uma grande variedade de presas, possibilitando descrever estratégias de forrageio e relacionar sua eficácia à disponibilidade de alimento, tipo de presa e sítio de alimentação (CROWLEY e LINTON, 1999).

A formiga-leão (Myrmeleon brasiliensis) é um inseto myrmeleontídeo pertencente à ordem Neuroptera. Esta família se caracteriza por seus integrantes serem predadores, tanto os adultos quanto as larvas, que constroem funis cônicos com diâmetros que podem variar de 2 e $5 \mathrm{~cm}$ e esperam por artrópodes de solo (por ex. formigas) que possam cair nesta armadilha (BORROR e DE LONG, 1988). A presa 
que cai no funil é detectada pela larva quando, ao tentar fugir, movimenta as paredes da armadilha e derruba grãos de areia no fundo do funil.

Já que a única maneira das larvas acessarem recursos alimentares é por meio da construção dos funis de areia (CROWLEY e LINTON, 1999), espera-se que exista um tamanho ótimo para o funil, proporcional ao tamanho da larva, onde os custos energéticos relacionados com sua construção não superem os ganhos alimentares, uma vez que o objetivo é acumular reservas energéticas para o estágio adulto (KRAMER, 2001).

As larvas de neurópteros e suas armadilhas são excelentes modelos de estudo de predação, já que podem ser comumente encontradas em substratos com granulosidade variada e consumirem uma grande variedade de presas, possibilitando descrever estratégias de forrageio e relacionar sua eficácia à disponibilidade de alimento, tipo de presa e sítio de alimentação (CROWLEY e LINTON, 1999). Desse modo, os objetivos deste estudo foram: (1) avaliar a relação entre o comprimento da larva de formiga-leão e o diâmetro do funil e (2) relacionar a probabilidade de predação ao diâmetro do funil.

\section{MATERIAL E MÉTODOS}

A área de estudo está inserida no bioma Caatinga, que ocorre apenas no Brasil, cobrindo mais de $700 \mathrm{~km}^{2}$ na região nordeste (SCARDUA, 2004). O experimento foi conduzido na Estação Ecológica do Seridó com cerca de 1.128 ha, situada no município de Serra Negra do Norte, RN, em período seco (maio/2012). A vegetação predominantemente é do tipo secundária, com prevalência de espécies arbustivas e arbóreas de pequeno porte (BENCKE et al., 2006). A ocorrência de larvas de formigaleão não é restrita ao bioma Caatinga, mas a elevada taxa de evaporação anual no bioma favorece substratos secos, onde os funis de larvas de formigas-leão são preferencialmente construídos (MC CLURE, 1983).

O desenho experimental consistiu em dois transectos, cada um com 40 metros de comprimento. Ao longo destes, foram demarcados 12 quadrantes de 1 $\mathrm{m}^{2}$, em locais onde havia presença de armadilhas (funis) de formiga-leão. Em cada quadrante foram escolhidos aleatoriamente 4 armadilhas, sendo uma de cada classe de tamanho (muito pequeno, pequeno, médio e grande), onde eram lançadas as presas para a captura. As presas utilizadas consistiam de térmitas da casta operária de Nasutitermes sp. (Isoptera: Termitidae), as quais eram lançadas no centro do funil. O tempo de captura foi medido com um cronômetro digital e definido como o intervalo em segundos entre os eventos de aterrissagem das presas no substrato e a captura pelas larvas. Eventos de captura após 30 segundos da aterrissagem não foram considerados. 
Em paralelo, foram coletadas 12 larvas de formiga-leão na área de amostragem, sendo medidos com paquímetro digital os comprimentos da larva e sua respectiva armadilha.

As análises foram realizadas no programa SYSTAT $12^{\circledR}$. Testamos através de uma regressão linear a relação entre o comprimento total das larvas e o diâmetro de seus respectivos funis e a influência do diâmetro do funil no tempo de captura das presas. Utilizando uma regressão logística, avaliamos a probabilidade de predação em relação ao tamanho do funil. Adicionalmente, utilizamos uma ANOVA para testar se os diferentes observadores influenciaram nas respostas das larvas.

\section{RESULTADOS}

Foram contabilizados em média $31 \pm 14$ armadilhas $/ \mathrm{m}^{2}$ (Mínimo = 14; Máximo=52), com diâmetro variando de 28,5 a $63,5 \mathrm{~mm}$. O comprimento das larvas variou de 8,6 a $10,8 \mathrm{~mm}$.

Encontramos uma relação positiva entre o comprimento da larva e o diâmetro da armadilha ( $R^{2}=0,792 ; \mathrm{p}=0,011 ; \mathrm{n}=12$, equação 1) (Figura 1).

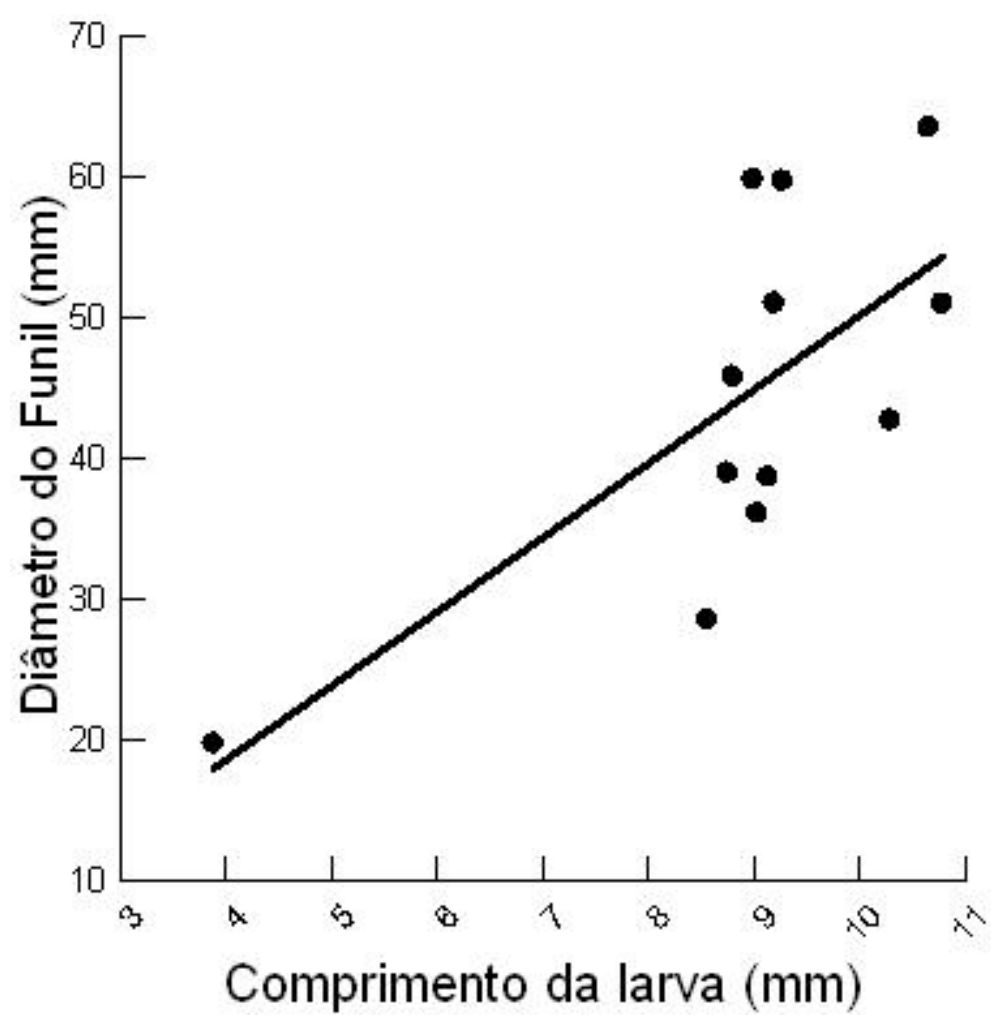

Figura 1: Efeito do comprimento da larva (mm) no tamanho da armadilha (mm).

$\mathrm{DF}=-2,474+5,264^{*} \mathrm{CL}$ (equação 1)

Onde: $\mathrm{DF}=$ Diâmetro do funil e $\mathrm{CL}=$ Comprimento da larva. 
A probabilidade da predação se relacionou negativamente com o diâmetro da armadilha (Odds ratio = 0,932 (o,878; 0,989; p = o,o12) (Figura 2).

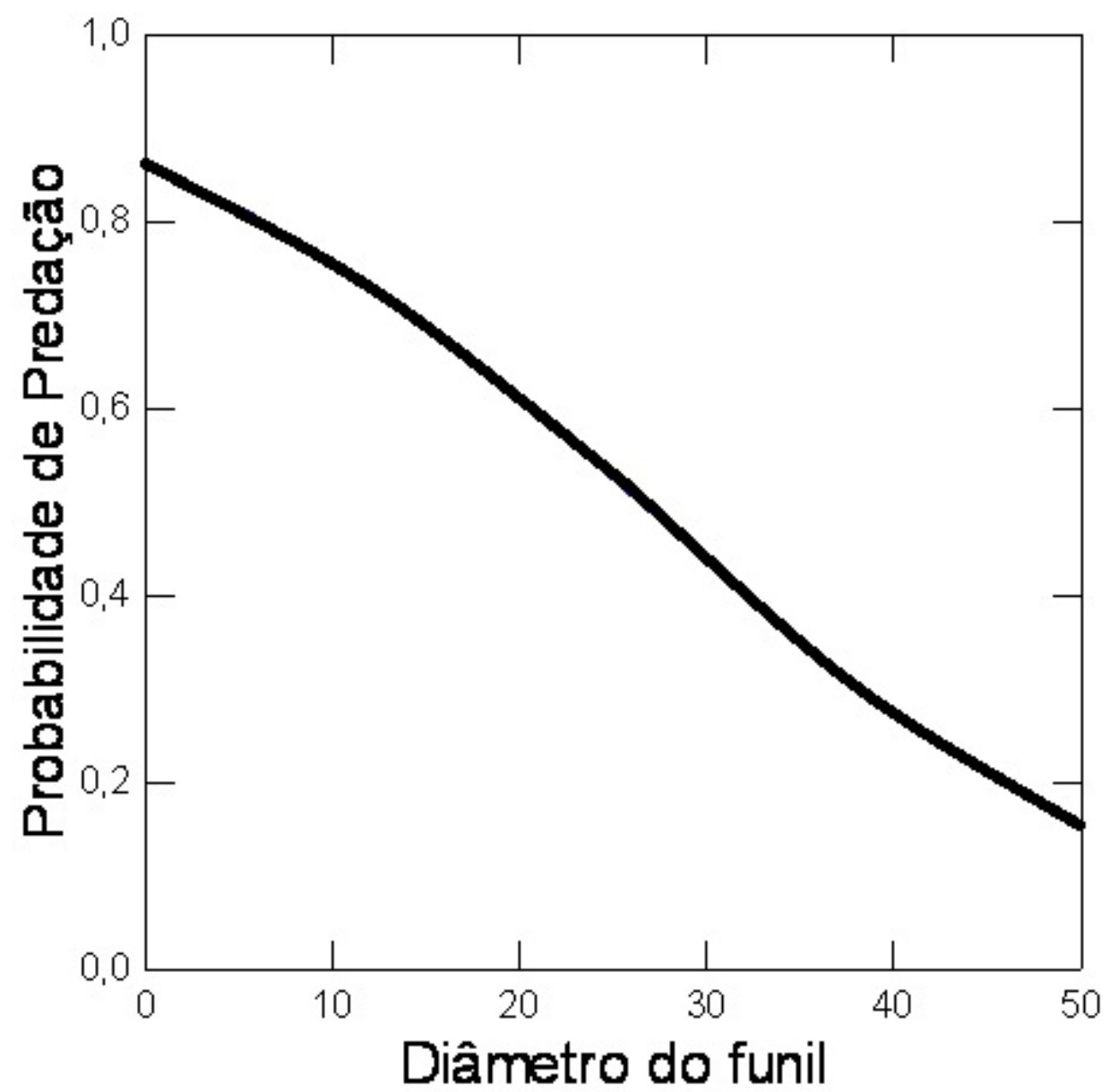

Figura 2: Relação entre diâmetro do funil e probabilidade de predação.

Não foram encontradas diferenças significativas entre a identidade do observador e o tempo de reposta $(\mathrm{p}=0,44 ; \mathrm{F}=0,937, \mathrm{~N}=26)$ ou probabilidade de predação $(\mathrm{p}=0,409 ; \mathrm{F}=0,985, \mathrm{n}=48)$.

\section{DISCUSSÃO}

Nós demonstramos nesse estudo que maiores comprimentos de larvas de formiga-leão estão relacionadas a armadilhas também de maior tamanho, mas isso não reflete necessariamente em maior probabilidade de predação de térmitas. LIMA e FARIA (2007) encontraram uma relação positiva entre os tamanhos da larva e da armadilha, relação esta corroborada por nossos resultados apesar do pequeno número de amostras. Sendo assim, pudemos considerar que os diâmetros de armadilha amostrados são relacionados aos comprimentos das larvas, ou seja, armadilhas maiores foram construídas por larvas maiores. 
Tem sido sugerido por PES et al. (2002), que o aumento do funil deve aumentar a eficiência de captura, pois com a área da armadilha maior, a probabilidade de cair mais presas torna-se maximizada. Entretanto, ao contrário do proposto na literatura, nós encontramos que maiores armadilhas estão relacionadas a menor probabilidade de predação. $O$ fato de que larvas maiores (com tamanho proporcional a sua armadilha) têm menor probabilidade de atacar presas em potencial pode ser explicado pelo conceito de forrageio ótimo (BEGON et al., 2006), onde organismos maiores podem não ter seus gastos energéticos compensados ao atacar presas pequenas demais, já que ao utilizar apenas cupins operários a diferença entre presas foi minimizada. Assim, a resposta positiva da probabilidade de predação em função do tamanho da armadilha, deve ser verificada quando o tamanho das presas possam oferecer recursos alimentares com ganhos energéticos. De fato, DIAS et al. (2006) não encontraram relação direta entre o diâmetro do funil e tempo de captura, mas larvas maiores ainda mostraram maior eficiência de captura. Nossos dados sugerem que, se a disponibilidade de recursos é restrita aos térmitas, a maior probabilidade de captura ocorrerá quanto menor for a abertura da armadilha de formiga-leão.

A ausência de efeito significativo do observador na contagem do tempo de captura da presa e na probabilidade de ocorrência de captura indica que os diferentes observadores não influenciaram os resultados.

Os dados desse estudo apontam para importantes estratégias no comportamento de predação de Neuroptera, entretanto, trabalhos futuros precisam ser desenvolvidos avaliando outras variedades de recursos (presas) para contribuir no entendimento do comportamento deste gênero, sobretudo em regiões semi-áridas onde o comportamento de muitas espécies ainda não está totalmente elucidado.

\section{AGRADECIMENTOS}

O primeiro autor agradece a FAPERN pela bolsa de estudos. Os demais autores agradecem a CAPES pela concessão da bolsa de estudos. Agradecemos a Gindomar Gomes pela orientação, a Carlos Fonseca pela ajuda no delineamento amostral e a todos os demais professores e funcionários da ESEC Seridó pela ajuda logística e intelectual.

\section{REFERÊNCIAS}

BEGON, M.; TOWNSEND, C. e HARPER, J.L. 2006. Ecology: from Individuals to Ecosystems. Blackwell Publishing, Oxford. 
BENCKE, G. A.; MAURÍCIO, G. N.; DEVELEY, P. F. e GOERCK, J. M. (orgs.). 2006. Áreas Importantes para a Conservação das Aves no Brasil. Parte I - Estados do Domínio da Mata Atlântica. São Paulo: SAVE Brasil.

BORROR, D. J. e DELONG, D.M. 1988. Introdução ao estudo dos insetos. Editora Edgard Blücher Ltda. São Paulo. 653 p.

CORTEZ, A.J.S.; CORTEZ, P. H. M.; FRANCO, J. M. e UZUNIAN, A. 2007. Caatinga. Editora Harbra, Coleção Biomas do Brasil. 64 pp.

CORWLEY, P. H.; e LINTON, M. C. 1999. Antlion foraging: tracking prey across space and time. Ecology 8o(7): 2271-2282.

DIAS, S. C.; SANTOS, B. A.; WERNECK, F. P; LIRA, P. K.; CARRASCO-CARBADILLO, V. e FERNANDES, G. W. 2006. Efficiency of prey subjugation by one species de Myrmeleon larvae (Neuroptera: Myrmeleontidae) in the Central Amazonia. Brazilian Journal of Biology, Notes and comments 66(2A): 441-442.

LIMA, T. N. e FARIA, R. R. 2007. Seleção de microhabitat por larvas de formiga-leão Myrmeleon brasilienses (Návas) (Neuroptera: Myrmeleontidae), em uma reserva florestal, Aquidauana, MS. Neotropical Entomology, 36(5): 812-814.

LUCAS, J. R. 1985. Metabolic rates and pit-construction costs of two antlion species. Journal of Animal Ecology, 54:295-309.

KRAMER, D. L. 2001. Foragin behavior; pp 232-246. In: FOX, C. W.; ROFF, D. A. e FAIRBAIRN, D. J. (Eds.), Evolutionary Ecology Concepts and Case Studies. Oxford: Oxford University Press.

MCCLURE, M. S. 1983. Myrmeleon (Hormiga León, Antlions). pp 742-743. In: JANZEN D.H. (Ed.), Costa Rican Natural History. The University of Chicago Press, London.

PES, A. M. O.; PIMENTEL, F.; JORGE, M. L.; VEIGA, M. C. e HIDALGO, M. 2002. Larvas de formigas-leão (Myrmeleontidae, Neuroptera) famintas aumentam o esforço de captura? pp 115-120. In: VENTICINQUE, E.; ZUANON, J. (Eds.), Ecologia da Floresta Amazônica. PDBFF/INPA, Manaus.

REYNOLDS, S. E.; NOTTINGHAM, S. F. e STEPHENS, A. E. 1985. Food and water economy and its relation to growth in fifth-instar larvae of the tobacco hornworm, Manduca sexta. Journal of Insect Physiology, 31:119-127. 
REYNOLDS, S. E. 1990. Feeding in caterpillars: maximizing or optimizing food acquisition? pp 106-118. In: MELLINGER, J. (Ed.), Animal Nutrition and Transport Processes. Basel, Karger.

RICKLEFS, R. E. 2010. A Economia da Natureza. $6^{\circ}$ edição, Guanabara Koogan, Rio de Janeiro, Brasil. 545 p.

TOWNSEND, C. R.; BEGON, M. e HARPER, J. L. 2003. Essentials of Ecology. Blackwell Science, Malden. 